\title{
Frequency-Hopping Transmitter Fingerprint Feature Classification Based on Kernel Collaborative Representation Classifier
}

\author{
Ping Sui, Ying Guo, Kun-feng Zhang, and Honguang Li \\ Institute of Information and Navigation, Air Force Engineering University, Xian, Shaanxi 710077, China \\ Correspondence should be addressed to Ping Sui; ziwuningxin@163.com
}

Received 15 June 2017; Accepted 27 August 2017; Published 9 October 2017

Academic Editor: Donatella Darsena

Copyright (C) 2017 Ping Sui et al. This is an open access article distributed under the Creative Commons Attribution License, which permits unrestricted use, distribution, and reproduction in any medium, provided the original work is properly cited.

\begin{abstract}
Noncooperation frequency-hopping (FH) transmitter fingerprint feature classification is a significant but challenging issue for FH transmitter recognition, since not only is it sensitive to noise but also it has the nonlinear, non-Gaussian and nonstability characteristics, which make it difficult to guarantee the classification in the original signal space. To address these problems, a method of frequency-hopping transmitter fingerprint feature classification based on kernel collaborative representation classifier is proposed in this paper. First, the noise suppression pretreatment of the FH transmitter signal is carried out by using the wave atoms frame method. Then, the nuances of the FH transmitters in the feature space are characterized by the surrounding-line integral bispectra features. And finally, incorporating the kernel function, a classifier which can generalize a linear algorithm to nonlinear counterpart is constructed for the final transmitter fingerprint feature classification. Extensive experiments on real-world FH transmitter "turn-on" transient signals demonstrate the robust classification of our method.
\end{abstract}

\section{Introduction}

Frequency-hopping $(\mathrm{FH})$ signals are generated by varying the carrier frequencies according to a certain hopping pattern, which is typically pseudo-random. Due to their inherent capability of low interception, good confidentiality, and strong anti-interference, FH signals have become an important tactical means of antireconnaissance and anti-jamming in military communication.

FH transmitter identification is a traditional significant but challenging issue in the electromagnetic war domain, especially under serious noise and noncooperation conditions. Due to the individual nuances of $\mathrm{FH}$ transmitters, there exist the inherent features, which can be used to identify the transmitter individuals. This inherent feature based on the individual nuances can be called the fingerprint of the transmitters.

Benefiting from the nuances among the transmitters, a number of studies using transient response features were emphatically researched to achieve the identification. Xu [1] combined the maximum correlation processing with time-dependent statistical method to realize the FH signal recognition. Eric et al. [2] proposed the MUSIC method based on signal direction information to sort the FH signals. Luo et al. [3] used the transient response feature of the transmitting power amplifiers to obtain the recognition results. However, when it comes to the condition of serious noise and complicated electromagnetic environment, especially when the transmitters are noncooperative, the practical classification of such methods is not ideal. In recent years, with the advantage of insensitivity to noise and outperform of classification, some methods based on sparse representation have been rapidly developed in various fields of signal classification. In the early studies, the sparse representation of the training data can be found via the $l_{0}$ minimization problem. However, this problem is proved to be an NP-hard problem [4]. Fortunately, theoretical results show that if the sparsely code solution of the training data is sparse enough, then the solution of $l_{1}$ and $l_{0}$-minimization problems is equivalent $[5,6]$. Therefore, Wright et al. [7] proposed a sparse representation-based classifier (SRC), in which sparse representation problem can be addressed by 
the $l_{1}$-minimization optimization. Although there are lots of fast numerical algorithms that have been proposed for the $l_{1}$-minimization optimization, it is still very computationally demanding. In contrast, Zhang et al. [8] argued both theoretically and empirically that collaborative representation classifier (CRC) based on the $l_{2}$-minimization is significantly more computationally efficient and can result in similar performance compared with the SRC. And also Yang et al. [9] proposed a relaxed collaborative representation model, which is simple but very competitive with the state-of-the-art classification method. Thus, CRC has been widely used for the research of signal classification, and a recent study has shown that CRC is more efficient than the SRC without sacrificing the classification accuracy. However, CRC is conducted in the original signal space rather than the nonlinear high dimensional feature space; thus the effectiveness of CR for the classification is difficult to be guaranteed in the original signal space when it is used to describing the nonlinear, non-Gaussian and nonstability feature of the FH transmitter fingerprints.

Above all, there are still some issues that have not yet been properly addressed, in particular, (1) the influence of noise. Fingerprints of the $\mathrm{FH}$ transmitter are so subtle and seriously sensitive to noise; (2) effectiveness of classification: a recent study has shown that $\mathrm{CR}$ is a promising regularization framework for signal classification. However, it is conducted in the original signal space rather than the nonlinear high dimensional feature space so that the effectiveness of the classification is difficult to be guaranteed.

To address these issues, in this paper, an effective $\mathrm{FH}$ transmitter fingerprint feature classification method based on kernel collaborative representation classifier is proposed. Firstly, we denoise the signal data by wave atoms frame instead of the traditional wavelet-based method. Secondly, utilizing the surrounding-line integral bispectra analysis, we extract the fingerprint features of the FH transmitters by their real-world "turn-on" transient signals. Finally, the kernel collaborative representation classifier by introducing the kernel function was used to realize the classification results. The main advantages of our method include the following: (1) the data noises are especially inevitable in electric environment. Our noise suppression method based wave atoms frame outperforms the traditional wavelet-based denoising method; (2) instead of the CRC, which is conducted in the original signal space, our method generalizes the linear CRC to its nonlinear counterpart by using the kernel function, and in this way, the fingerprint features belonging to the same class can be easily separated. Experiments on real-world FH "turnon" transient signals demonstrate the effectiveness and high classification of our method.

The rest of this paper is organized as follows. The theories of wave atoms frame and CRC are expounded in Section 2. Then Section 3 describes the procedures of noise suppression and the details of the proposed KCRC method. Section 4 demonstrates the experimental results and analyzes the classification performance. Finally, Section 5 concludes the paper.

\section{Preliminaries}

This section starts with a succinct description of the wave atoms and offers insight into its implementation. And then some previous works in classification are introduced. In this part, we present the traditional sparse representation method first and then give the collaborative representation-based classifier and its deficiencies.

2.1. Wave Atoms Frame. In a general signal processing problem, the wave atoms are represented as $\phi_{\mu}(x)$, with subscript $\mu=(j, \mathbf{m}, \mathbf{n})=\left(j, m_{1}, m_{2}, n_{1}, n_{2}\right)$, where $j, m_{1}, m_{2}, n_{1}, n_{2} \in Z$ and index is a point $\left(x_{\mu}, w_{u}\right)$ in phase-space as

$$
\begin{aligned}
x_{\mu} & =2^{-j} \mathbf{n}, \\
w_{u} & =\pi 2^{j} \mathbf{m}, \\
C_{1} 2^{-j} & \leq \max _{i=1,2}\left|m_{i}\right| \leq C_{2} 2^{j},
\end{aligned}
$$

where $C_{1}, C_{2}>0$ are two constants, the position vector $x_{\mu}$ is the center of $\phi_{\mu}(x)$, and the wave vector $w_{u}$ determines the centers of both bumps of $\widehat{\phi}_{\mu}(x)$ as $\pm w_{u}$ in frequency plane.

Let $x_{u}$ and $w_{u}$ be as in (1) for some $C_{1}, C_{2}>0$. The elements of a frame of wave packets $\left\{\phi_{\mu}\right\}$ are called wave atoms for all $M>0$ when

$$
\begin{aligned}
\left|\phi_{\mu}(x)\right| \leq & C_{M} \cdot 2^{j}\left(1+2^{j}\left|x-x_{\mu}\right|\right)^{-M} \\
\left|\widehat{\phi}_{\mu}(w)\right| \leq & C_{M} \cdot 2^{-j}\left(1+2^{j}\left|w-w_{\mu}\right|\right)^{-M}+C_{M} \\
& \cdot 2^{-j}\left(1+2^{-j}\left|w+w_{\mu}\right|\right)^{-M} .
\end{aligned}
$$

Consider the localization condition; one-dimensional wave atoms can be obtained by constructing the frequency domain tightly support symmetric $\widehat{\psi}_{m}^{0}(w)$; and deal with it by two-dimensional scaling and translation as follows [10]:

$$
\begin{gathered}
\widehat{\psi}_{m}^{0}(w)=e^{-i w / 2}\left[e^{i \alpha_{m}} g\left(\varepsilon_{m}\left(w-\pi\left(m+\frac{1}{2}\right)\right)\right)\right. \\
\left.+e^{-i \alpha_{m}} g\left(\varepsilon_{m+1}\left(w+\pi\left(m+\frac{1}{2}\right)\right)\right)\right],
\end{gathered}
$$

where $\varepsilon_{m}=(-1)^{m}, \alpha_{m}=(\pi / 2)(m+1 / 2)$. The function $g$ is an appropriate real-valued, $C^{\infty}$ bump function with a support interval length of $2 \pi$ and it satisfied $\sum_{m}\left|\widehat{\psi}_{m}^{0}(w)\right|^{2}=1$. And by combining dyadic dilates and translating $\widehat{\psi}_{m}^{0}$ on the frequency axis one-dimensional wave atoms can be noted as

$$
\psi_{m, n}^{j}(x)=\psi_{m}^{j}\left(x-2^{-j} n\right)=2^{j / 2} \psi_{m}^{0}\left(2^{j} x-n\right) .
$$

To preserve the orthonormality of the $\psi_{m, n}^{j}(x)$, the profile $g$ needs to be asymmetric in addition to all the other properties, in the sense that $g(-2 w-\pi / 2)=g(\pi / 2+w)$ for $w \epsilon$ $[-\pi / 3, \pi / 3]$, with $g$ itself being supported on $[-7 \pi / 6,5 \pi / 6]$. 
Considering $H$ is a Hilbert transform, the orthogonal basis and its dual orthogonal basis can be defined as

$$
\begin{aligned}
& \phi_{\mu}^{+}\left(x_{1}, x_{2}\right)=\psi_{m_{1}}^{j}\left(x_{1}-2^{-j} n_{1}\right) \psi_{m_{1}}^{j}\left(x_{2}-2^{-j} n_{2}\right) \\
& \phi_{\mu}^{-}\left(x_{1}, x_{2}\right)=H \psi_{m_{1}}^{j}\left(x_{1}-2^{-j} n_{1}\right) H \psi_{m_{1}}^{j}\left(x_{2}-2^{-j} n_{2}\right) .
\end{aligned}
$$

It is easy to see that the recombination

$$
\begin{aligned}
& \phi_{\mu}^{(1)}=\frac{\phi_{\mu}^{+}+\phi_{\mu}^{-}}{2}, \\
& \phi_{\mu}^{(2)}=\frac{\phi_{\mu}^{+}-\phi_{\mu}^{-}}{2}
\end{aligned}
$$

provides basic functions with two bumps in the frequency plane, symmetric with respect to the origin, and, hence, the directional wave packets. Together $\phi_{\mu}^{(1)}$ and $\phi_{\mu}^{(2)}$ form the wave atom frame and may be denoted jointly as $\phi_{\mu}$. If the frame is tight, there are

$$
\sum_{\mu}\left|\left\langle\phi_{\mu}^{(1)}, \mu\right\rangle\right|^{2}+\sum_{\mu}\left|\left\langle\phi_{\mu}^{(2)}, \mu\right\rangle\right|^{2}=\|u\|^{2}
$$

Then we have the two-dimensional wave atoms transform coefficient as [10]

$$
C_{\mu}=\left\langle\phi_{\mu}^{(1)}, \mu\right\rangle+\left\langle\phi_{\mu}^{(2)}, \mu\right\rangle .
$$

2.2. Collaborative Representation-Based Classifier. Assume that the training data set is represented as $\mathbf{A}=\left[\mathbf{A}_{1}, \mathbf{A}_{2}\right.$, $\left.\ldots, \mathbf{A}_{C}\right] \in \mathbf{R}^{d \times N}$, where $C$ is the number of classes, $\mathbf{A}_{i}=$ $\left[a_{1}^{i}, a_{2}^{i}, \ldots, a_{n_{i}}^{i}\right] \in \mathbf{R}^{d \times n_{i}}$ is the collection of the data points for class $i$, and $N=\sum_{i=1}^{C} n_{i}$ is the total number of training data. Then the sparse representation of a new test data $\mathbf{y} \in \mathbf{R}^{d}$ can be found via the $l_{0}$-minimization problem as

$$
\begin{aligned}
\widehat{\boldsymbol{\alpha}}=\arg \min _{\boldsymbol{\alpha}} & \|\boldsymbol{\alpha}\|_{0} \\
\text { s.t. } & \mathbf{y}=\mathbf{A} \boldsymbol{\alpha},
\end{aligned}
$$

where $\|\cdot\|_{0}$ denotes the $l_{0}$-norm which is defined to be the number of nonzero elements in a vector. Generally, a sparse solution via the $l_{0}$-minimization is more robust and facilitates the consequent classification of the test data $\mathbf{y} \in \mathbf{R}^{d}$. However, this problem (9) is proved to be an NP-hard problem [4]. Fortunately, theoretical results show that if the solution $\widehat{\boldsymbol{\alpha}}$ obtained is sparse enough, then the solution of the $l_{0}$ and $l_{1}$-minimization problems is equivalent $[5,6]$. Therefore, in SRC [7], the test data $\mathbf{y} \in \mathbf{R}^{d}$ can be sparsely coded by the $l_{1}$-minimization problem as

$$
\begin{aligned}
\widehat{\boldsymbol{\alpha}}=\arg \min _{\boldsymbol{\alpha}} & \|\boldsymbol{\alpha}\|_{1} \\
\text { s.t. } & \mathbf{y}=\mathbf{A} \boldsymbol{\alpha} .
\end{aligned}
$$

Although the $l_{1}$-minimization problem is extensively studied and lots of numerical algorithms have been proposed, it is still a computationally demanded problem. In contrast,
Zhang et al. [8] verified both empirically and theoretically that $l_{2}$-minimization based classifier relying on collaborative representation can result in $\widehat{\boldsymbol{\alpha}}$ in a similar performance. The collaborative representation of $\mathbf{y} \in \mathbf{R}^{d}$ can be written as

$$
\begin{aligned}
\widehat{\boldsymbol{\alpha}}=\arg \min _{\boldsymbol{\alpha}} & \|\boldsymbol{\alpha}\|_{2}^{2} \\
\text { s.t. } & \mathbf{y}=\mathbf{A} \boldsymbol{\alpha} .
\end{aligned}
$$

Then the classifier based on collaborative representation is ruled as

$$
\operatorname{class}(y)=\arg \min _{i} \frac{\left\|\mathbf{y}-\mathbf{A}_{i} \widehat{\boldsymbol{\alpha}}\right\|_{2}}{\|\widehat{\boldsymbol{\alpha}}\|_{2}}, \quad i=1,2, \ldots, C .
$$

Compared with the $l_{1}$-minimization based sparse representation, extensive experimental results in [8] demonstrate that the $l_{2}$-minimization base collaborative representation is more computationally efficient.

\section{Proposed Method}

As for FH transmitters, there will be many actual and significant transient states during its work. Some of these transitions are from the system itself, such as "turn-on/off" instantaneous changes and mode switch of the transmitters; others are from the outside interference, which is accidental and does not exist for every transmitter. And these transitions which reflect the characteristics of the transient states contain a wealth of individual nuances information of the FH transmitters. Based on the analysis above, in order to fully characterize individual nuances of the FH transmitters, we choose the instantaneous "turn-on" response of the $\mathrm{FH}$ signals to calculate the fine features for the final transmitter classification.

3.1. Noise Suppression. The FH signals collected in the actual environment often have noise and clutter interference. In order to effectively reduce the influence of external disturbances on the feature extraction of transient signals, our method performs the noise reduction pretreatment on the collected transient signals firstly.

At the present time, one-dimensional signal noise suppression is mainly based on the wavelet analysis. Due to the nonstationarity of the transient signals of FH transmitter, the traditional wavelet-based denoising algorithms can reduce some noise, but they blur the signal information at the same time. In contrast, experimental results in [11] demonstrate that wave atoms frame is more significantly denoising efficient without sacrificing more signal accuracy. Therefore, in this paper, we use the wave atoms frame to carry out the denoising result of the "turn-on" transient FH signal.

Let $s(t)$ be the source "turn-on" signal, and $n(t)$ the additive noise, which follows the Gaussian distribution, and also they are uncorrelated; then we have the observed signal as

$$
x(t)=s(t)+n(t) .
$$

Wave atoms frame is a special two-dimensional wave pocket deformation and is usually used for image and other 
two-dimensional signal denoising. Thus, we construct a virtual observation matrix $\mathbf{X}$ for the $x(t)$ by adding white noise.

$$
\begin{aligned}
\mathbf{X} & =\left[\begin{array}{c}
x_{1}(t) \\
x_{2}(t) \\
\vdots \\
x_{P}(t)
\end{array}\right]=\left[\begin{array}{c}
x(t) \\
x(t)+n_{2}(t) \\
\vdots \\
x_{P}(t)+n_{P}(t)
\end{array}\right] \\
& =\left[\begin{array}{c}
s(t)+n(t) \\
s(t)+n(t)+n_{2}(t) \\
\vdots \\
s(t)+n(t)+n_{P}(t)
\end{array}\right],
\end{aligned}
$$

where $n(t), n_{2}(t), \ldots, n_{P}(t)$ obey the Gaussian distribution. In matrix $\mathbf{X}$, the signal between the lines is determined by the nuances characteristics of the $\mathrm{FH}$ transmitter; the information correlation is strong at all times. When $n(t)$ is random noise, the correlation is weak.

Sampling the virtual observation matrix $\mathbf{X}$ at $[0, t]$, the number of sampling points is $L$; obtain its discrete form $\widehat{\mathbf{X}}=$ $\left[\widehat{\mathbf{X}}_{1 \times L}, \widehat{\mathbf{X}}_{2 \times L}, \ldots, \widehat{\mathbf{X}}_{P \times L}\right]$ as

$$
\widehat{\mathbf{X}}=\left[\begin{array}{cccc}
x_{1}\left(\frac{t}{L}\right) & x_{1}\left(\frac{2 t}{L}\right) & \cdots & x_{1}(t) \\
x_{2}\left(\frac{t}{L}\right) & & \\
\vdots & \ddots & \\
x_{P}\left(\frac{t}{L}\right) & \cdots & & x_{P}(t)
\end{array}\right]
$$

In this way, the matrix $\widehat{\mathbf{X}}$ can be treated as a twodimensional signal matrix for noise reduction. The effective information in $\widehat{\mathbf{X}}$ is equivalent to the vertical texture feature of the two-dimensional image. And then we can use the superiority of wave atoms frame for two-dimensional texture information expression to obtain the denoising result $\widehat{\mathbf{X}}^{*}$; the final denoising one-dimensional signal can be written as

$$
\mathbf{x}=\frac{1}{P} \sum_{i=1}^{P} \widehat{\mathbf{X}}_{i \times L}^{*}
$$

3.2. Feature Extraction. Compared with the traditional firstorder and second-order spectrums, high-order spectrum can extract more significant features of nonstationary, nonGaussian and nonlinear signals. In this paper, we extract the surrounding-line integral bispectra features to characterize the fingerprints of the $\mathrm{FH}$ transmitters in the feature space. The bispectrum of noise suppression data $\mathbf{x}$ is defined as

$$
\begin{aligned}
& B_{\mathbf{x}}\left(\omega_{1}, \omega_{2}\right) \\
& \quad=\int_{-\infty}^{+\infty} \int_{-\infty}^{+\infty} c_{3 \mathbf{x}}\left(\tau_{1}, \tau_{2}\right) e^{-j\left(\omega_{1} \tau_{1}+\omega_{2} \tau_{2}\right)} d \tau_{1} d \tau_{2},
\end{aligned}
$$

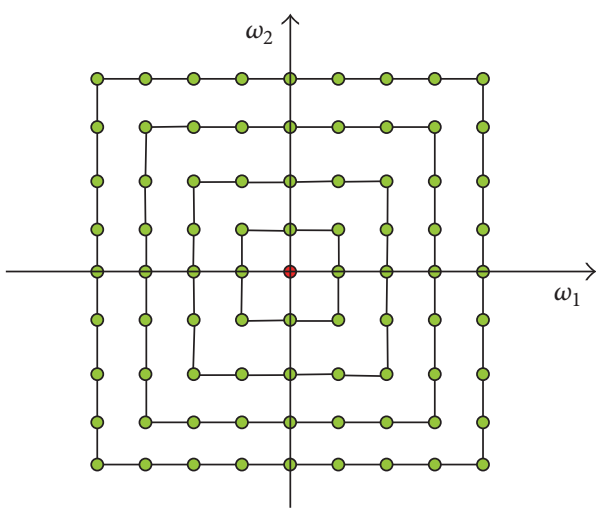

FIGURE 1: The integral paths of integral bispectra.

where $c_{3 \mathbf{x}}\left(\tau_{1}, \tau_{2}\right)$ is the three-order cumulant of $\mathbf{x}$. After obtaining the bispectrum, we use the surrounding-line integral bispectra analysis method to process the bispectral estimation result. As shown in Figure 1, the integral path is a square centered around the origin, and each point represents a bispectral estimate.

This integral path does not miss out or reuse any bispectrum values, which ensure the integrity of the target information. Furthermore, this calculation transforms the results from two dimensions into one dimension, reducing the computationally complexity [11]. And finally, datasets of fingerprint characteristics are established by the surroundingline integral bispectra features $B_{\mathbf{x}}\left(\omega_{1}, \omega_{2}\right)$.

3.3. Kernel Collaborative Representation Classifier. In the machine learning field, the kernel function is a well-known technique, which can generalize a linear algorithm to its nonlinear counterpart in which features belonging to the same class are better grouped together and thus are easily classified. Assume there is a nonlinear feature mapping function $\Phi(\cdot): R^{d} \rightarrow R^{D}(d \ll D)$. It transforms the test data $\mathbf{y} \in \mathbf{R}^{d}$ and the training dictionary $\mathbf{A}=\left[\mathbf{A}_{1}, \mathbf{A}_{2}, \ldots, \mathbf{A}_{C}\right] \epsilon$ $\mathbf{R}^{d \times N}$ to their high dimensional feature space: $\mathbf{y} \rightarrow \Phi(\mathbf{y}), \mathbf{A}=$ $\left[\mathbf{A}_{1}, \mathbf{A}_{2}, \ldots, \mathbf{A}_{C}\right] \rightarrow \Phi(\mathbf{A})=\left[\Phi\left(\mathbf{A}_{1}\right), \Phi\left(\mathbf{A}_{2}\right), \ldots, \Phi\left(\mathbf{A}_{C}\right)\right]$. Then we get the kernel collaborative representation classifier (KCRC)

$$
\begin{aligned}
\widehat{\boldsymbol{\alpha}}=\arg \min _{\boldsymbol{\alpha}} & \|\boldsymbol{\alpha}\|_{2}^{2} \\
\text { s.t. } & \Phi(\mathbf{y})=\Phi(\mathbf{A}) \boldsymbol{\alpha},
\end{aligned}
$$

where $\Phi(\cdot)$ is typically unknown and can only accessed by kernel function $k(\mathbf{x}, \mathbf{y})=\langle\Phi(\mathbf{x}), \Phi(\mathbf{y})\rangle=\Phi^{T}(\mathbf{x}) \Phi(\mathbf{y})$. In this paper, we take advantage of the Gaussian kernel PCA algorithm to solve the optimization problem in (18) due to its excellent performance reported in the paper [12-15]. The Gaussian kernel can be written as

$$
k(\mathbf{x}, \mathbf{y})=\exp \left(-\gamma\|\mathbf{x}-\mathbf{y}\|^{2}\right)
$$

Suppose there is a transformation matrix $\mathbf{P}$ which projects the high dimensional data points into a low dimensional subspace with dimensionality $m$.

$$
\mathbf{P}=\Phi(\mathbf{A}) \mathbf{B},
$$




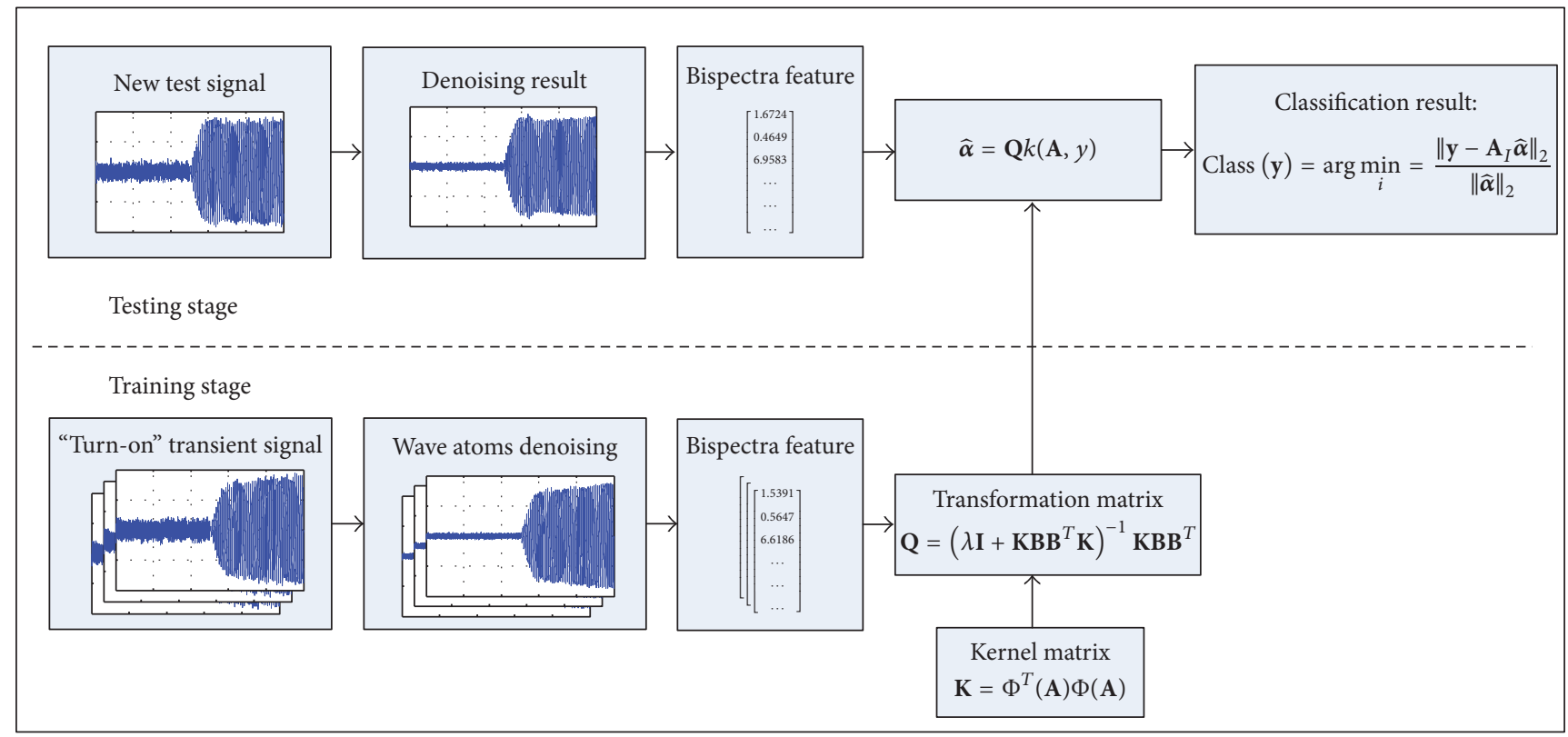

FIGURE 2: The transmitter fingerprint feature classification process of our method.

where $\mathbf{B}=\left[\boldsymbol{\beta}_{1}, \boldsymbol{\beta}_{2}, \ldots, \boldsymbol{\beta}_{m}\right] \in \mathbf{R}^{N \times m}, \boldsymbol{\beta}_{j}, j=1,2, \ldots, m(m<$ $N)$ is a normalized eigenvector associated with the $j$ th largest eigenvalues corresponding to the kernel matrix $\mathbf{K}=$ $\Phi^{T}(\mathbf{A}) \Phi(\mathbf{A})$.

By introducing the transformation matrix $\mathbf{P}$ to Eq. (14), we can get

$$
\begin{aligned}
\widehat{\boldsymbol{\alpha}}=\arg \min _{\boldsymbol{\alpha}} & \|\boldsymbol{\alpha}\|_{2}^{2} \\
\text { s.t. } & \mathbf{P}^{T} \Phi(\mathbf{y})=\mathbf{P}^{T} \Phi(\mathbf{A}) \boldsymbol{\alpha} .
\end{aligned}
$$

Let $\lambda>0$ be a Lagrange parameter and introduce the Lagrangian to (21), the object function of KCRC can be rewritten as follows:

$$
\begin{aligned}
L(\boldsymbol{\alpha})= & \left\|\mathbf{P}^{T} \Phi(\mathbf{y})-\mathbf{P}^{T} \Phi(\mathbf{A}) \boldsymbol{\alpha}\right\|_{2}^{2}+\lambda\|\boldsymbol{\alpha}\|_{2}^{2} \\
= & \Phi^{T}(\mathbf{y}) \mathbf{P} \mathbf{P}^{T} \Phi(\mathbf{y})-\Phi^{T}(\mathbf{y}) \mathbf{P} \mathbf{P}^{T} \Phi(\mathbf{A}) \boldsymbol{\alpha} \\
& -\boldsymbol{\alpha}^{T} \Phi^{T}(\mathbf{A}) \mathbf{P} \mathbf{P}^{T} \Phi(\mathbf{y}) \\
& +\boldsymbol{\alpha}^{T} \Phi^{T}(\mathbf{A}) \mathbf{P} \mathbf{P}^{T} \Phi(\mathbf{A}) \boldsymbol{\alpha}+\lambda \boldsymbol{\alpha}^{T} \boldsymbol{\alpha} .
\end{aligned}
$$

The optimal solution to (22) requires

$$
\begin{aligned}
\frac{\partial L}{\partial \boldsymbol{\alpha}} & =\lambda \boldsymbol{\alpha}+\Phi^{T}(\mathbf{A}) \mathbf{P P}^{T} \Phi(\mathbf{A}) \boldsymbol{\alpha}-\Phi^{T}(\mathbf{A}) \mathbf{P P}^{T} \Phi(\mathbf{y}) \\
& =0 .
\end{aligned}
$$

Substituting (20) into (23), we have

$$
\widehat{\boldsymbol{\alpha}}=\mathbf{Q} k(\mathbf{A}, \mathbf{y}),
$$

where $k(\mathbf{A}, \mathbf{y})=\Phi^{T}(\mathbf{A}) \Phi(\mathbf{y})$ and $\mathbf{Q}=\left(\lambda \mathbf{I}+\mathbf{K B B}^{T} \mathbf{K}\right)^{-1}$. $\mathbf{K B B}^{T}$. Clearly, $\mathbf{Q}$ is independent of $\mathbf{y}$ such that it could be precalculated, which reduces the computational complexity and thus should be more efficient.

The procedures of FH transmitter classification based on kernel collaborative representation classifier are illustrated in Figure 2. In the training stage, we first extract the surrounding-line integral bispectra features $\mathbf{A}$ of the noise suppressed "turn-on" transient FH signals and then calculate the transformation matrix $\mathbf{Q}$. In the testing stage, given a new test signal $\mathbf{y}$, the corresponding operation of noise suppression and feature extraction is applied, and then the target is classified as one of the known FH transmitters based on the KCRC.

\section{Experimental Results}

In this section, experiments are conducted on 100 records of training signal for each of $5 \mathrm{FH}$ transmitters. And all experiments are implemented in Matlab 2014a and run on a PC with Intel Core i7, 2.93 GHz CPU and 4 GB RAM.

4.1. Noise Robustness. In order to verify the noise robustness of our method, we compared the recognition rate with whether or not to produce denoising, the recognition results of the classifier are shown in Figure 2, the red line is the experimental result using the training data samples which have been preproduced by wave atoms frame based noise suppression, and the other line is the experimental result without noise suppression. From Figure 3, we can see that our wave atoms frame based noise suppression method overcomes the influences of noise and performs high recognition rate in the experiment. Figure 4 shows the signal denoising results by our method and wavelet-based method; from the red circle in this figure we can clearly find that our method outperforms 


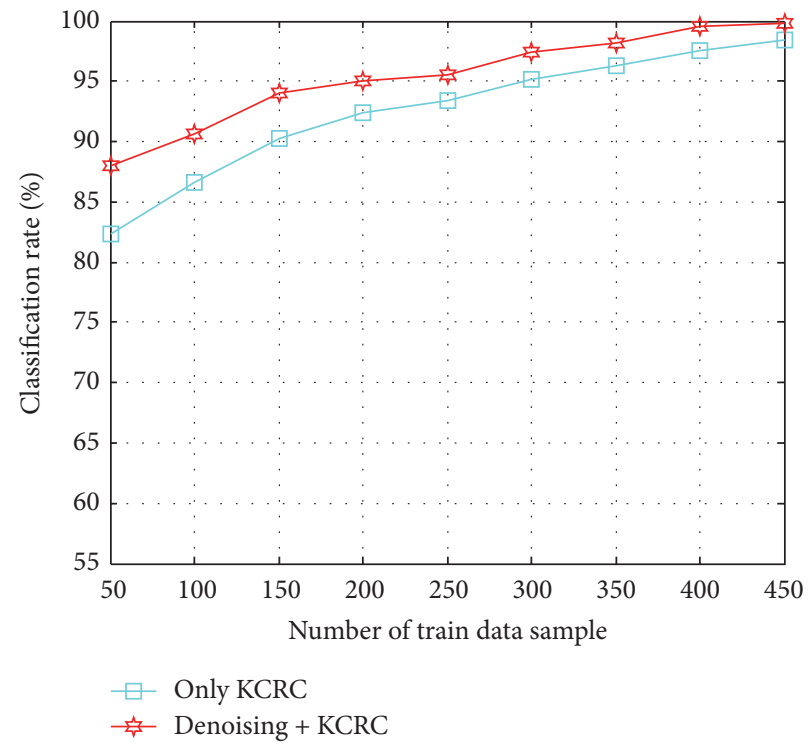

Figure 3: Classification rate with whether or not to produce denoising.
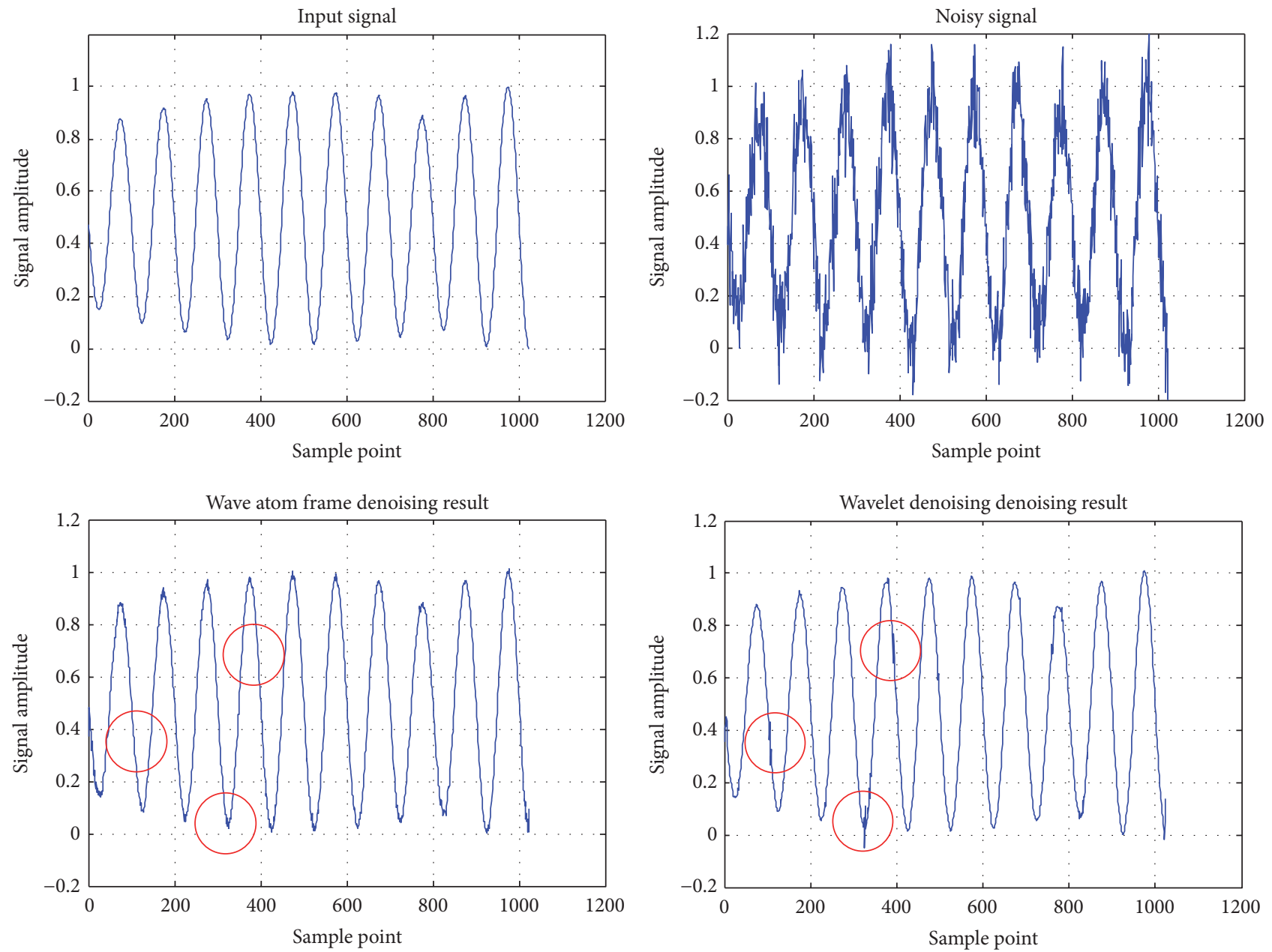

Figure 4: Denoising result with different algorithms. 

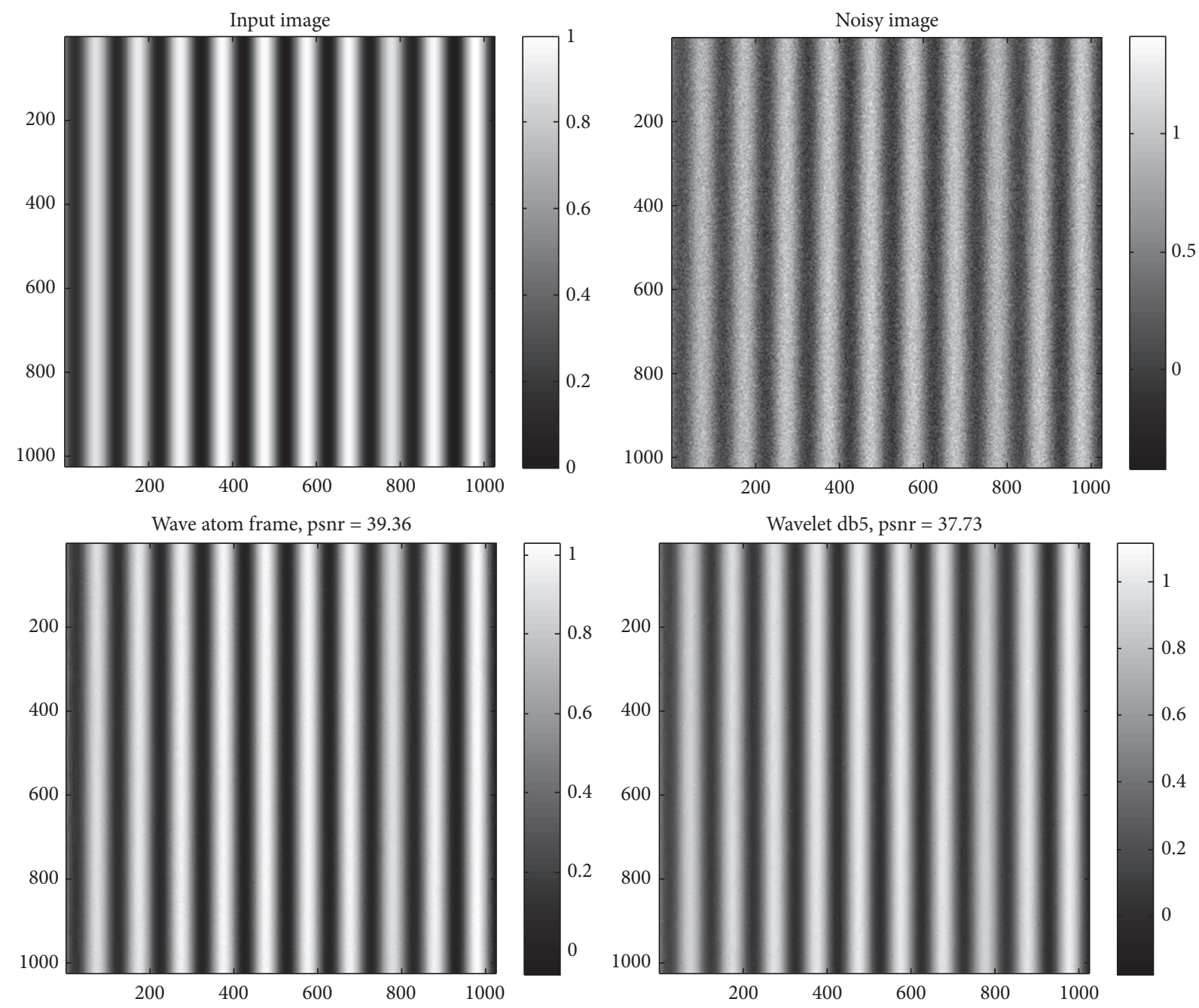

Figure 5: Two-dimensional displays of signals in Figure 3.

the state-of-the-art wavelet-based denoising algorithm, and Figure 5 shows the two-dimensional display corresponding to the signals in Figure 4.

4.2. Effectiveness of Classification. Classification efficiency is important for real-world $\mathrm{FH}$ transmitter classification application. As illustrated in Figure 2 the proposed method mainly requires a kernel function to generalize a linear algorithm to its nonlinear counterpart in which the accuracy of classification could be ensured. We compare the classification efficiency of our method with KNN, SVM, SRC, and CRC; the results are illustrated in Table 1 and Figure 6. From this experiment we could conclude that our method always shows a powerful classification ability compared with other classifiers.

Furthermore, for evaluating the proposed algorithm comprehensively, we also compared the methods of KNN, SVM, SRC, and CRC with signals of the original, wavelet treated, and wave atoms treated, respectively. As seen from Table 2 and Figure 7, the wave atoms frame denoised result

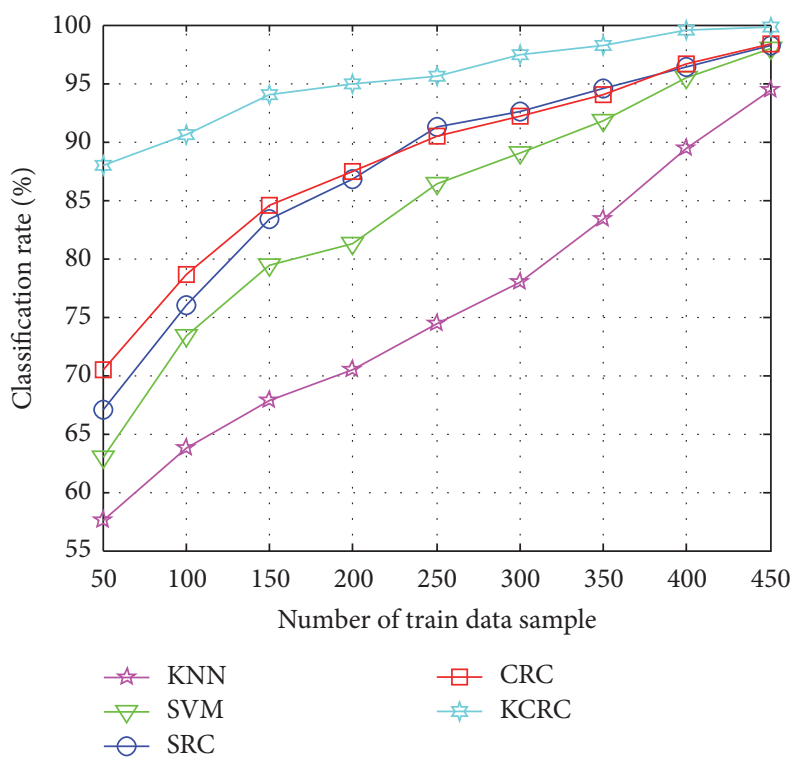

FIgURE 6: The classification rate with different method. 


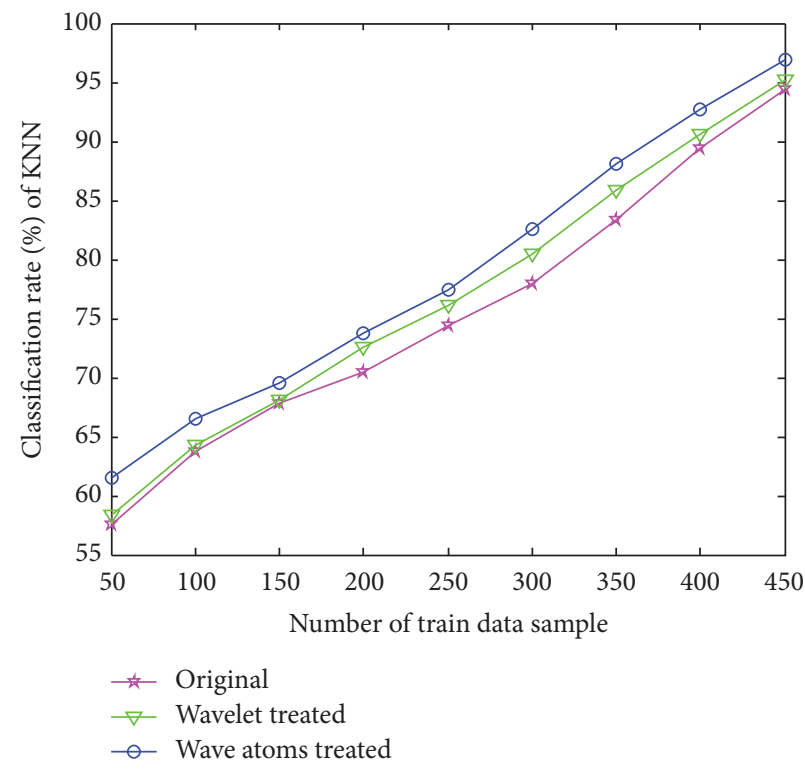

(a) Classification result of $\mathrm{KNN}$

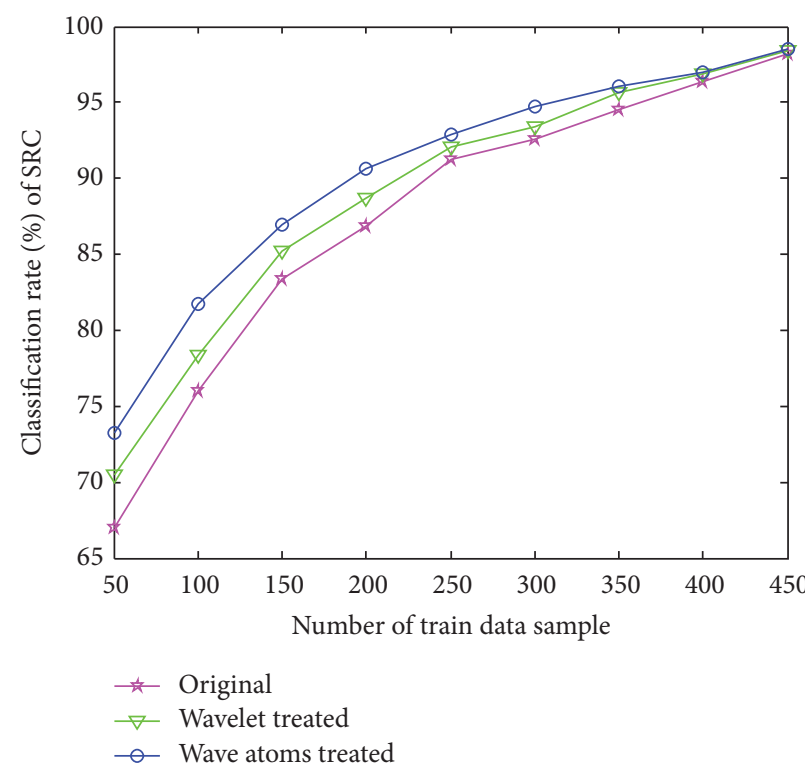

(c) Classification result of SRC

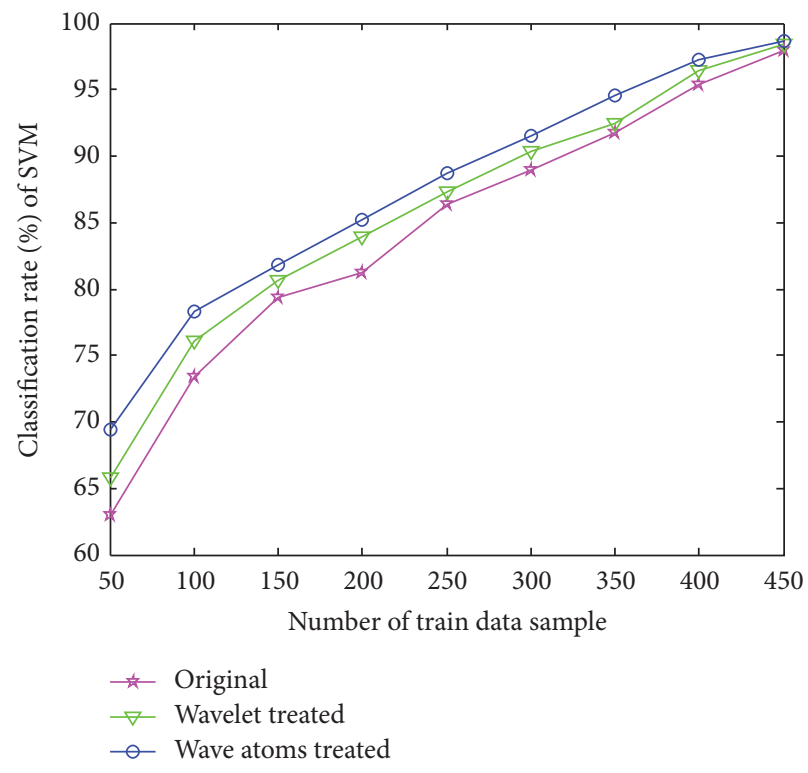

(b) Classification result of SVM

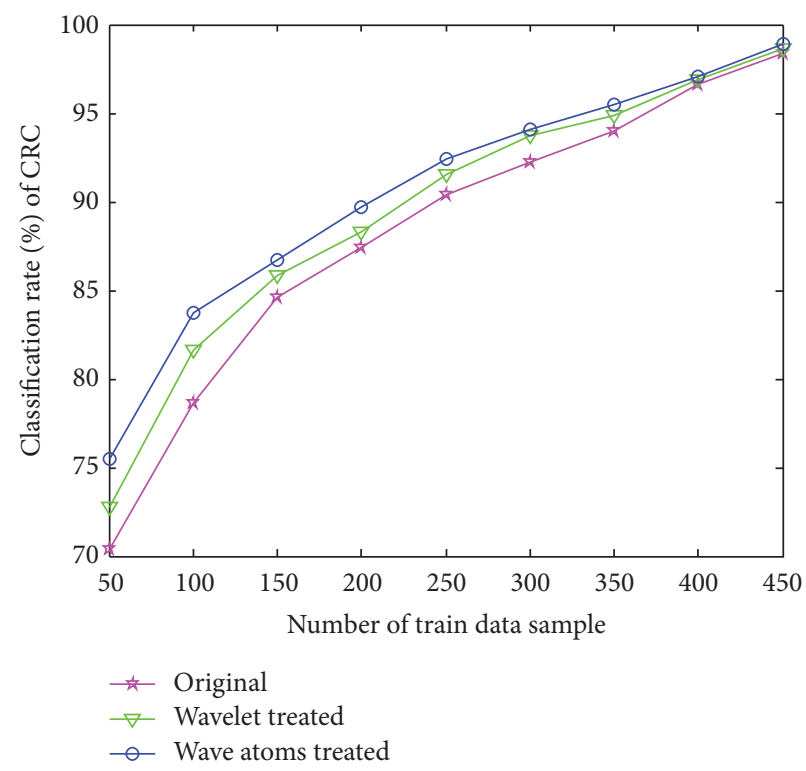

(d) Classification result of CRC

FIGURE 7: The classification rate of different treated signals with the methods of KNN, SVM, SRC, and CRC.

TABLE 1: The classification rate with different methods (\%).

\begin{tabular}{lccccc}
\hline Number of train sample & KNN & SVM & SRC & CRC & KCRC \\
\hline 50 & 57.6 & 63.0 & 67.0 & 70.4 & 88.0 \\
100 & 63.8 & 73.4 & 76.0 & 78.6 & 90.6 \\
150 & 67.8 & 79.4 & 83.4 & 84.6 & 94.0 \\
200 & 70.4 & 81.2 & 86.8 & 87.4 & 95.0 \\
250 & 74.4 & 86.4 & 91.2 & 90.4 & 95.6 \\
300 & 78.0 & 89.0 & 92.6 & 92.2 & 97.4 \\
350 & 83.4 & 91.8 & 94.5 & 94.0 & 98.2 \\
400 & 89.4 & 95.4 & 96.4 & 96.6 & 99.6 \\
450 & 94.4 & 98.0 & 98.2 & 98.4 & 99.8 \\
\hline
\end{tabular}

has the best performance in all experiments. It demonstrates that our wave atoms frame noise reduction pretreatment has significant contribution to the final identification result.

\section{Conclusion}

In this paper, a novel method based on kernel collaborative representation is proposed for $\mathrm{FH}$ transmitter fingerprint feature classification. Firstly, our method takes advantage of the wave atoms based noise suppression algorithm to remove the influence of noise; thus the accuracy and efficiency of the fingerprint feature extraction are improved. And then our method utilizes the surrounding-line integral bispectra 
TABLE 2: The classification rate of different treated signals (\%).

\begin{tabular}{|c|c|c|c|c|c|c|c|c|c|}
\hline Number of train sample & 50 & 100 & 150 & 200 & 250 & 300 & 350 & 400 & 450 \\
\hline \multicolumn{10}{|l|}{ KNN } \\
\hline Original & 57.6 & 63.8 & 67.8 & 70.4 & 74.4 & 78.0 & 83.4 & 89.4 & 94.4 \\
\hline Wavelet & 58.4 & 64.3 & 68.1 & 72.6 & 76.1 & 80.4 & 85.9 & 90.6 & 95.2 \\
\hline Wave atoms & 61.5 & 66.5 & 69.6 & 73.8 & 77.5 & 82.6 & 88.1 & 92.7 & 96.9 \\
\hline \multicolumn{10}{|l|}{$S V M$} \\
\hline Original & 63.0 & 73.4 & 79.4 & 81.2 & 86.4 & 89.0 & 91.8 & 95.4 & 98.0 \\
\hline Wavelet & 65.8 & 76.1 & 80.6 & 83.9 & 87.3 & 90.3 & 92.4 & 96.4 & 98.4 \\
\hline Wave atoms & 69.4 & 78.3 & 81.8 & 85.2 & 88.7 & 91.5 & 94.6 & 97.2 & 98.7 \\
\hline \multicolumn{10}{|l|}{$S R C$} \\
\hline Original & 67.0 & 76.0 & 83.4 & 86.8 & 91.2 & 92.6 & 94.5 & 96.4 & 98.2 \\
\hline Wavelet & 70.5 & 78.4 & 85.2 & 88.7 & 92.1 & 93.4 & 95.6 & 96.9 & 98.4 \\
\hline Wave atoms & 73.2 & 81.7 & 87.0 & 90.6 & 92.9 & 94.7 & 96.1 & 97.0 & 98.5 \\
\hline \multicolumn{10}{|l|}{$C R C$} \\
\hline Original & 70.4 & 78.6 & 84.6 & 87.4 & 90.4 & 92.2 & 94.0 & 96.6 & 98.4 \\
\hline Wavelet & 72.8 & 81.6 & 85.8 & 88.3 & 91.5 & 93.7 & 94.9 & 96.9 & 98.6 \\
\hline Wave atoms & 75.5 & 83.7 & 86.7 & 89.7 & 92.4 & 94.1 & 95.5 & 97.1 & 98.9 \\
\hline
\end{tabular}

features of "turn-on" transient signals more effectively by introducing a kernel function to generalize the linear algorithm to its nonlinear counterpart. Experimental results on real-world $5 \mathrm{FH}$ transmitters show that our method achieves obviously better performance than CRC and several state-ofthe-art methods in terms of accuracy and efficiency.

\section{Conflicts of Interest}

The authors declare that there are no conflicts of interest regarding the publication of this paper.

\section{Acknowledgments}

This research was supported by the National Natural Science Foundation of China (no. 61601500).

\section{References}

[1] Q. H. Xu, Recognition Method for Frequency-Hopping in Signal Reconnaissance, Xidian University, Xian, China, 2005.

[2] M. Eric, M. L. Dukic, and M. Obradovic, "Frequency-hopping signal separation by spatio-frequency analysis based on Music method," in Proceedings of The Sixth International Symposium on Spread Spectrum Techniques and Applications, vol. 66 of 82, 78 pages, 2000

[3] Z. X. Luo, Z. J. Zhao, and J. Y. Xu, "Individual Frequencyhopping transmitter identification based on transient response of power amplifier," Journal of Hangzhou Dianzi University, vol. 29, no. 1, pp. 29-32, 2009.

[4] E. Amaldi and V. Kann, "On the approximability of minimizing nonzero variables or unsatisfied relations in linear systems," Theoretical Computer Science, vol. 209, no. 1-2, pp. 237-260, 1998.

[5] D. L. Donoho, "For most large underdetermined systems of linear equations the minimal $l_{1}$-norm solution is also the sparsest solution," Communications on Pure and Applied Mathematics, vol. 59, no. 6, pp. 797-829, 2006.
[6] E. J. Candes and T. Tao, "Near-optimal signal recovery from random projections: universal encoding strategies?" Institute of Electrical and Electronics Engineers. Transactions on Information Theory, vol. 52, no. 12, pp. 5406-5425, 2006.

[7] J. Wright, A. Y. Yang, A. Ganesh, S. S. Sastry, and Y. Ma, "Robust face recognition via sparse representation," IEEE Transactions on Pattern Analysis and Machine Intelligence, vol. 31, no. 2, pp. 210-227, 2009.

[8] L. Zhang, M. Yang, and X. Feng, "Sparse representation or collaborative representation: Which helps face recognition?" in Proceedings of the IEEE International Conference on Computer Vision (ICCV '11), pp. 471-478, Barcelona, Spain, November 2011.

[9] M. Yang, L. Zhang, D. Zhang, and S. Wang, "Relaxed collaborative representation for pattern classification," in Proceedings of the IEEE Conference on Computer Vision and Pattern Recognition (CVPR '12), pp. 2224-2231, IEEE, Providence, RI, USA, June 2012.

[10] L. Demanet and L. Ying, "Wave atoms and sparsity of oscillatory patterns," Applied and Computational Harmonic Analysis. Time-Frequency and Time-Scale Analysis, Wavelets, Numerical Algorithms, and Applications, vol. 23, no. 3, pp. 368-387, 2007.

[11] Z. Tang and Y. K. Lei, "Radio transmitter identification based on collaborative representation," Wireless Personal Communications, pp. 1-15, 2017.

[12] M. Yang, "Kernel eigenfaces vs. kernel fisherfaces: face recognition using kernel method," in proceedings of the IEEE International Conference on Automatic Face and Gesture Recognition, pp. 215-220, 2008.

[13] V. N. Vapnik, Statistical Learning Theory, Wiley-Interscience, New York, NY, USA, 1998.

[14] B. Schölkopf, A. Smola, and K.-R. Müller, "Nonlinear component analysis as a kernel eigenvalue problem," Neural Computation, vol. 10, no. 5, pp. 1299-1319, 1998.

[15] B. Wang, W. Li, N. Poh, and Q. Liao, "Kernel collaborative representation-based classifier for face recognition," in Proceedings of the 2013 38th IEEE International Conference on Acoustics, Speech, and Signal Processing, ICASSP 2013, pp. 2877-2881, Vancouver, Cannada, May 2013. 


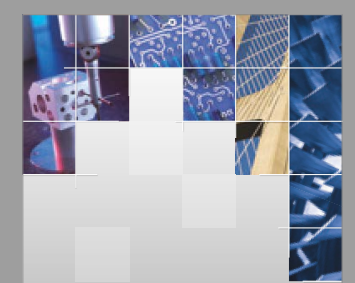

\section{Enfincering}
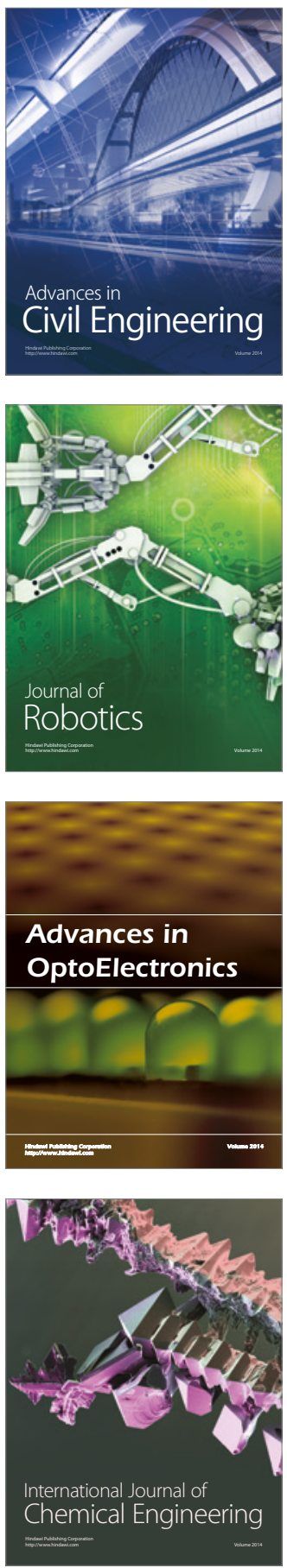

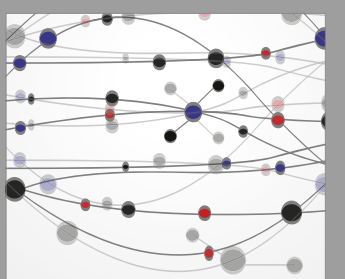

The Scientific World Journal

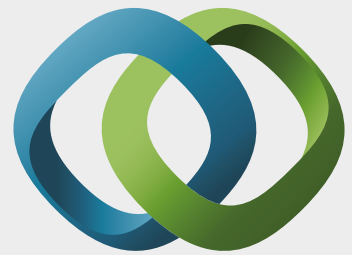

\section{Hindawi}

Submit your manuscripts at

https://www.hindawi.com
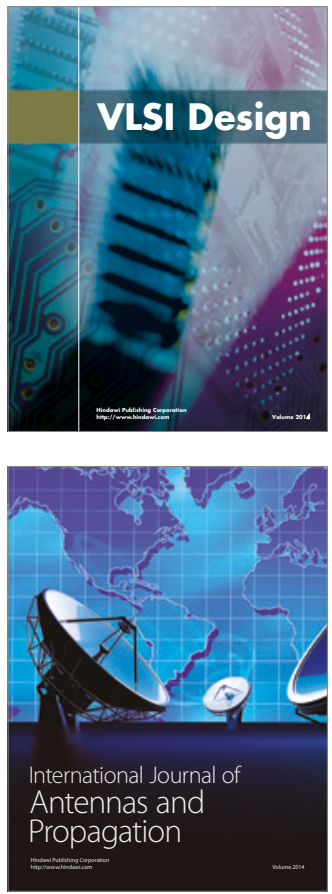

\section{Rotating}

Machinery
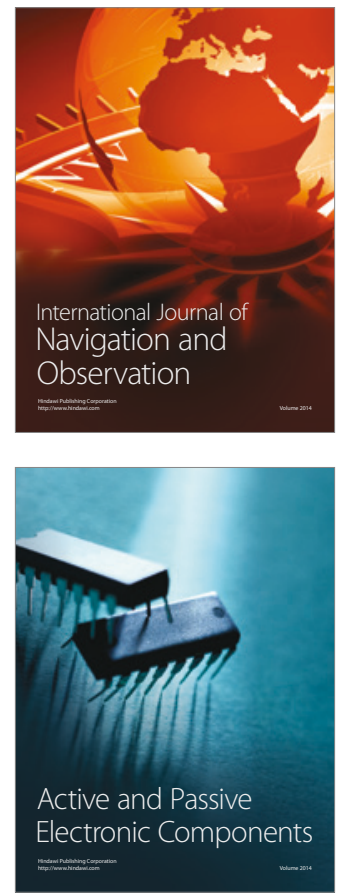
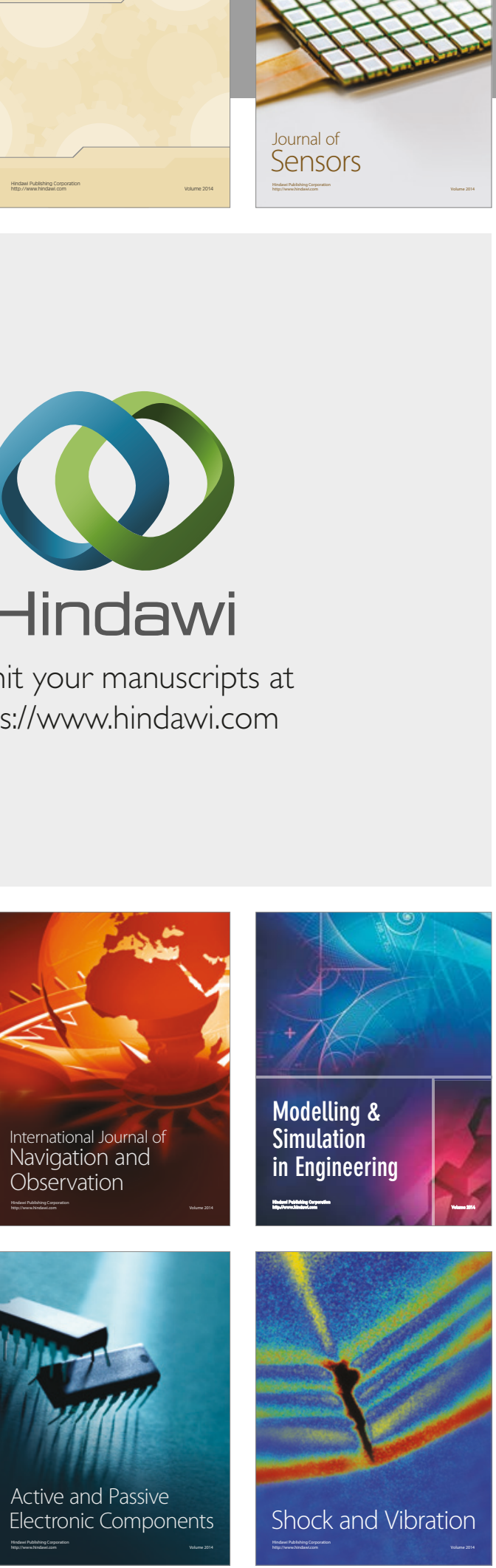
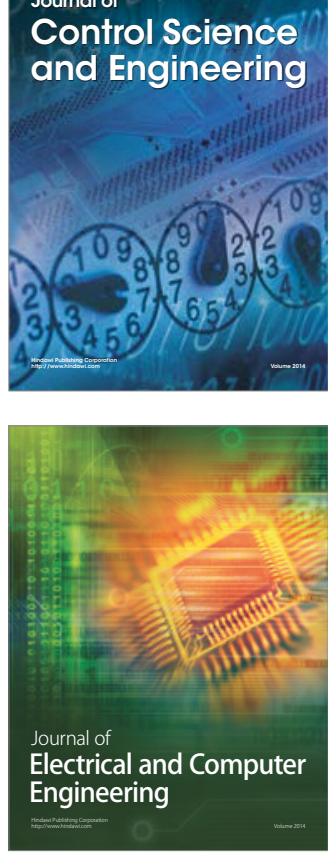

Distributed

Journal of

Control Science

and Engineering
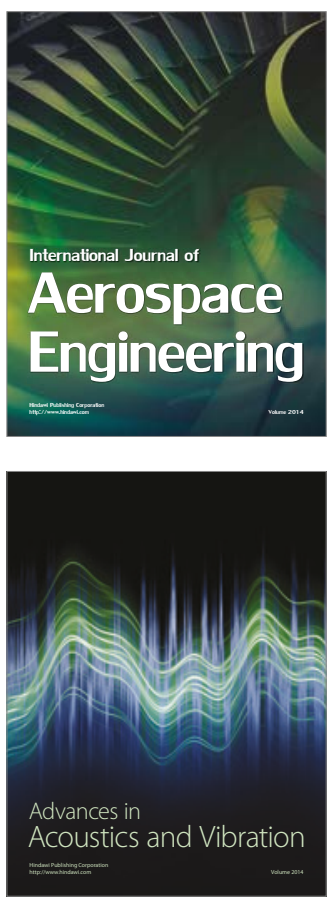

Sensor Networks 